\title{
INVESTIGACIÓN/RESEARCH
}

Recibido: 21/01/2014 ---Aceptado: 18/02/2015 ---Publicado: 15/03/2015

\section{LA GENTE MAYOR COMO SUJETO Y OBJETO DE LA TELEVISIÓN. EL ESTUDIO EN ANDALUCÍA}

Agustín Olmo López¹. Universidad de Sevilla. España.

aolmo@uses

José Antonio Navarro Moreno². Universidad de Sevilla. España.

janava@us.es

\section{RESUMEN}

Este trabajo trata de desvelar la doble relación que existe entre las personas mayores y la televisión. Se da por supuesto que son unos grandes consumidores de la misma, puesto que la televisión juega un papel importante en su tiempo libre, pero está menos analizado cómo y cuánto aparece la tercera edad como objeto de una noticia. Así, nos hemos centrado en buscar los programas informativos de una televisión concreta, en este caso la de Andalucía, durante una temporada completa para fijarnos cuántas veces aparecen y cuál ha sido el contenido de los temas en que intervienen. Así hemos visto que son poco tratados, menos de lo que cuantitativamente significa este grupo de edad en la población, y hemos detallado los temas en que son más recogidos. Vemos que en los programas diarios se centran más en su relación con

\footnotetext{
${ }^{1}$ Agustín Olmo López: Doctor en Periodismo, periodista en Canal Sur TV y profesor asociado de la Universidad de Sevilla. Está especializado en periodismo audiovisual.
}

\footnotetext{
${ }^{2}$ José Antonio Navarro Moreno: Doctor en Periodismo, periodista en Canal Sur TV y profesor asociado de la Universidad de Sevilla. Especializado en Relaciones Públicas y Gabinetes de Comunicación.
} 
asuntos derivados de la política, mientras que en los programas no diarios, son más utilizados como sujetos activos.

Descriptores: Tercera edad, envejecimiento, televisión, mayores.

\section{OLD PEOPLE AS SUBJECT AND OBJECT IN TELEVISION. THE STUDY IN ANDALUCIA}

This work tries to reveal the double way in the relation between the elderly people and the television. It is known that they are big consumers because television has an important role in their free time, but is less analyzed how and how much they appear like object of news. Then we have analyzed all the news of a season of a particular television, - Andalucia Television named Canal Sur-, to evaluate how many times appear and which the approach of the topic has been. We have seen that appear less than quantitatively this age group is in the population, and have detailed the topics that are more common. We see that in the daily programs there are more in relation with political topics, while in not daily programs, they are more used like active subjects.

Keywords: old people, ageing, television, elder, news

\section{INTRODUCCIÓN}

$\mathrm{Si}$ aceptamos las previsiones demoscópicas, nuestra sociedad se dirige hacia el envejecimiento y en los últimos tramos de edad, un lugar importante de su actividad lo ocupa el tiempo destinado al ocio. No obstante, no hay que relacionar el grupo de edad de mayores (de 65 años en adelante), con un modelo único de comportamiento, puesto que es heterogéneo, como señala Requejo (1998:147) pues las personas son seres de transformación y no de adaptación. 
Podríamos recurrir a Paulo Freire (1997:74) para decir que no se es viejo o joven en función de cuándo uno nace, sino en función de cómo entendemos el mundo y de la curiosidad por saber.

El envejecimiento humano, apuntaba Díaz Casanova (1989:86), está universalmente entendido como un proceso de continua evolución psicológica, física y social a lo largo de la vida. Por tanto, no se puede admitir el estereotipo de un envejecimiento entendido solamente como deterioro o merma de facultades. Es evidente que se producen algunas disminuciones físicas, pero es más importante considerarlo como un proceso continuo de cambio ante todo tipo de nuevas situaciones.

Ese cambio se ha visto afectado por la tecnología pues ha impuesto en poco tiempo una transformación radical en la que se ha pasado del «homo sapiens» al «homo digitalis», como ya definía Terceiro (1996:27) hace casi 20 años. Ahí podría darse una discriminación negativa pues podría haber una brecha digital entre la generación de los mayores y los jóvenes.

Torres (2006:20) señala que el sentido de desarrollo personal variará con las generaciones que se incorporen al grupo de edad de mayores de 65 años. Lo que se defina hoy puede no ser válido para la siguiente generación, puesto que el desarrollo personal está basado en la realidad que ha construido la propia interacción social en la experiencia de cada generación. No es igual la experiencia de quien salió de la posguerra, que la de los nativos digitales.

Por eso, a la hora de reflexionar sobre la relación de las personas mayores con los medios de comunicación o analizar la imagen que ofrecen los medios sobre los mayores hemos recuperado el concepto definido por la UNESCO como envejecimiento activo (2002:12) entendiéndolo como el proceso de envejecimiento que permite a los mayores desarrollar su potencial físico, social y mental de manera aceptable, de acuerdo con sus necesidades y teniendo cuidados asistenciales cuando lo requieran. 
En general, los medios de comunicación, y en particular la televisión, juegan un papel importante para la tercera edad, como ocio, y como contenido, y como agenda de conversación y de pautas sociales. En todos los informes así aparece, pero la televisión no puede mantenerse como un simple mercado de ocio y entretenimiento, sino que es uno de los grandes enculturizadores, como Medrano y Cortés (2010:4) establecen.

\section{OBJETIVO}

El objetivo de este artículo es destacar descuido que existe en la televisión en general hacia un grupo de edad de población importante. Ni como consumidores de la misma, ni como objeto de sus contenidos se destaca su valor de forma proporcional a lo que cuantitativamente representa. Es más, incluso en las televisiones comerciales se acentúa esto al producirse casi un olvido de la tercera edad entendiendo que tiene poco interés como público al que dirigir su publicidad por su reducida capacidad adquisitiva, y por idéntica razón aparece menos en condición de protagonistas de noticias o series.

\section{METODOLOGÍA}

Destaca el escaso material que hemos encontrado actualizado en cuanto a la relación de tercera edad y medios de comunicación, y de hecho, para alguna referencia a la situación de este grupo de población hemos tenido que remontarnos a algún estudio que ya lleva bastantes años publicado, exceptuando los informes del Instituto para los Mayores y Servicios Sociales (IMSERSO). Tras ello hemos acudido a los datos estadísticos y a las encuestas publicadas por organismos oficiales como el Instituto Nacional de Estadística o el correspondiente andaluz para cuantificar el grupo de edad del que estamos hablando y su proporción en la población global, así como otros estudios sociológicos sobre sus hábitos, entre los que destacamos el consumo de medios de comunicación, especialmente la televisión.

Igualmente, hemos acudido al servicio de documentación de la televisión pública andaluza para hacer la búsqueda de las noticias que afectan a este sector de la población, para ver el contenido y el formato en que son presentadas. También, 
hemos contactado con el departamento de audiencias de la misma empresa para que nos facilitaran los datos de seguimiento de los programas informativos así como un desglose de los telespectadores por franja de edad.

Con todo ello hemos procedido a agrupar las noticias y reportajes en categorías generales de contenido semejante, y hemos estudiado los datos que ofrece como audiencia.

\section{ANÁLISIS Y RESULTADO}

\subsection{Población en la tercera edad}

La mayoría de las personas mayores piensa que ser mayor no depende de la edad. La respuesta dominante de los españoles es que una persona es mayor a los 70 años, según la Encuesta sobre Personas Mayores 2010 realizada por el IMSERSO.

Sin embargo, se suele asociar como umbral en el que una persona es considerada mayor los 65 años puesto que era la edad de jubilación hasta las últimas reformas. Si nos quedamos con ese dato de que población mayor es aquella que supera los 65 años, en España según el Instituto Nacional de Estadística (INE), son más de ocho millones de personas (casi el 17\% de la población). Además, el número de personas muy mayores -más de 80 años- se ha acentuado siendo más de dos millones y medio. (1)

En España entre 1992 y 2012, la esperanza de vida de los hombres ha pasado de 73,9 a 79,4 años y la de las mujeres de 81,2 a 85,1 años, según el INE. En sus Proyecciones de Población a Corto Plazo (10 años) y a Largo Plazo (40 años), se aprecia que si se mantienen los ritmos actuales la esperanza de vida alcanzaría 81,8 años en los hombres y 87,0 años en la mujeres en el año 2022; y 86,9 años para los hombres y de 90,7 para las mujeres en 2051.

\subsection{La tercera edad como contenido de las televisiones}

La relación entre la televisión y mayores tiene una doble perspectiva. La primera como grandes consumidores de la misma, y la segunda como contenidos de la misma. 
Si empezamos por esta última, hay poco publicado. Lo más completo, aunque limitado geográficamente, es un estudio de 2012 elaborado por el Consell de l'Audiovisual de Catalunya que analizaba la presencia de las personas mayores de 65 años en las televisiones de esa Comunidad (TV3, TVE en Cataluña, 8TV, y Barcelona TV), y detectaba que están representadas insuficientemente en el discurso televisivo pues aparecía un 7\% de personajes mayores en los programas de ficción, mientras que en los informativos tenía el 10,3\% de presencia en los noticiarios y un $13,6 \%$ en los debates y entrevistas, cuando en Cataluña es el 17,4\% de la población.

En relación con la programación de ficción el papel que tienen en la trama se debe, mayoritariamente, a su condición de personas jubiladas o de familiares otros personajes principales.

También, en el estudio de López y Cuenca sobre series de ficción españolas publicado en Comunicar en 2005, se ve que proyectan una imagen estereotipada de la tercera edad, con papeles de consejeros expertos y protectores de la familia. Predominan tres modelos aunque con matices, los de actores, colaboradores $u$ oponentes. Los dos primeros revestidos con rasgos de autoridad, mientras que como oponentes su actitud es la de quien no tiene nada mejor que hacer.

En menor medida, puesto que no era el objeto específico que se investigaba, el Consejo Audiovisual de Andalucía en 2011 publicó un informe sobre la presencia de los colectivos necesitados de mayor protección en los informativos de las televisiones públicas de la comunidad (Canal Sur, sus desconexiones, TVE en Andalucía y 10 operadores locales), y contabilizó 595 informaciones sobre tercera edad emitidas, lo que suponía el $0.51 \%$ de las noticias. Canal Sur TV fue la cadena que más noticias sobre este grupo de edad emitió (163). Los operadores dedicaron 13 horas 47 minutos y 48 segundos a informar sobre los mayores.

En el Libro Blanco sobre Envejecimiento Activo publicado por el IMSERSO denunciaba que para los medios de comunicación las personas mayores son invisibles y transmiten, en general, una imagen inadecuada porque lo abordan como un grupo homogéneo, cuando es tan heterogéneo como cualquier otro. Además, son marcados con estereotipos negativos, como protagonistas de sucesos, personas de salud muy deteriorada o beneficiarios de programas de ayudas. Con esta imagen sesgada y tópica, afianzan una percepción negativa del envejecimiento. 


\subsection{La tercera edad como consumidora de televisión}

Si el papel como contenido de la televisión está por debajo de su porcentaje de población, en cambio sí que destacan como grandes consumidores de la misma. Analizando los usos televisivos nos encontramos con una relación directa entre edad y consumo. La televisión se convierte en un elemento de compañía y socialización para los mayores. Observamos que, como el resto de la población, ha experimentado una subida en su consumo y se relaciona con la mayor oferta de canales con la TDT. Vaca (2004:88) decía que en 2004 el consumo de Televisión estaba en las cinco horas diarias, y diez años después supera las seis horas en algunos perfiles de mayores.

La encuesta del CIS de 2006 sobre condiciones de vida de las personas mayores recogía que el $90 \%$ ve la televisión todos o casi todos los días.

En la tesis doctoral sobre la relación de los mayores con la televisión en Málaga se destacaba que con sus pensiones pueden sufragar elementos de ocio, tienen una ideología muy asentada en sus convicciones, tienen una vida asociativa y de participación ciudadana y dialéctica, y su autor Montes (2012:235) indicaba que sus formatos preferidos son los clásicos de la televisión: noticias, películas antiguas y concursos, y que prefieren los informativos de Canal Sur por incidir en el entorno cercano.

Así, por primera vez nos encontramos con una generación de mayores, con calidad de vida, que se encuentra dentro de los criterios de envejecimiento activo y que la mayor parte de su vida ha convivido con la televisión.

Quizás por ello, vemos ejemplos paradójicos en España, pues mientras las televisiones públicas retiran de sus parrillas cierta programación barata con bajos niveles formativos que reproducen modelos sociales ajenos, como las telenovelas, otras cadenas comerciales las han recuperado acaparando parte de esta audiencia.

En este sentido, la sociedad debería plantearse si es posible la alfabetización mediática para adultos, para que estos espectadores tengan una actitud más activa 
frente al televisor y para ello, nada mejor que conseguir que los mismos medios, como ya recogía Aguaded (1999:162), se conviertan en la herramienta perfecta para el proceso de aprendizaje.

Una encuesta 'online', sobre el nivel de equipamiento tecnológico en los hogares de los mayores españoles, efectuado por E-Media, llevada a cabo por profesores de la Universidad Complutense de Madrid, indicaba que navegar por internet es ya un hábito más extendido entre los mayores de 65 años que comprar el periódico en el quiosco. También deja claro que todos los hogares en los que viven personas mayores cuentan con televisión mientras que, por ejemplo, un 22 \% carece de acceso a internet. Respecto al consumo de medios, el mayoritario es la televisión (informativos: 89\%; entretenimiento: $75 \%$ ).

Otro estudio de la Universidad Rey Juan Carlos en 2009 indicaba que los mayores ven la televisión 4 horas y media (270 minutos) al día solos o acompañados, y hasta 6 horas en el caso de que convivan con otras personas. El porcentaje llegaba hasta el 93\% sumando los que decían verla casi todos los días.

Además, un 37\% de los mayores de 65 años manifestaba poseer dos aparatos en su hogar. El estudio no sólo constata que ver la televisión es un hábito que se da especialmente entre los mayores, sino que obedece a unos patrones rutinarios.

Otro estudio del Consell de l'Audiovisual, al reflejar la dieta mediática en Catalunya, señalaba que si la población mayor de 45 años, consumía de media $4 \mathrm{~h} 40^{\prime}$ de televisión, $1 \mathrm{~h} 10^{\prime}$ de radio y 20' de diarios y revistas cada día; en la práctica, los mayores de 65 años destinaban ese mismo tiempo diario al consumo de medios de comunicación (6h y 20'), aunque la diferencia estribaba en que la gente mayor dedicaba aproximadamente $1 \mathrm{~h}$ menos a escuchar la radio y navegar por internet, $y$ este tiempo lo daba íntegramente a ver la televisión.

La población mayor es también la que prefiere seguir la televisión autonómica y local. En concreto, la penetración de la autonómica y de la local es un $23 \%$ más alto en la población mayor que en el conjunto de la población.

No es un fenómeno sólo de nuestro país. Un estudio realizado por la Universidad de California San Diego en 2011, en el que participaron 3.000 ciudadanos, concluía que a 
medida que se cumplían años se pasaba más tiempo ante el televisor, llegando incluso los mayores de edad a triplicar a otros grupos de edad.

En lo que se refiere al seguimiento según las cadenas, mientras en 2004 TVE tenía la audiencia más envejecida seguida de Antena 3, la televisión privada en los últimos años ha variado ligeramente su programación, rejuveneciendo su media.

La televisión autonómica, en cambio, ha mantenido altos niveles de población mayor siempre.

En nuestro estudio somos conscientes de la amplitud que supone el concepto de persona mayor y la relación que mantiene el grupo con los medios de comunicación, pues como asegura Ferrés (2005:238) un mismo producto audiovisual produce efectos distintos en función de diversas variables: la edad del espectador, la frecuencia del consumo, la personalidad, la ideología y el contexto en que se produce el consumo. Pues bien, en este grupo tenemos dos variables claramente definidas: edad y frecuencia.

Hasta hace unos años no se había considerado a las personas mayores como un grupo de consumidores importante al que había que ofertar productos y servicios específicos, sin embargo, el informe de la Fundación Edad y Vida (2009:54) señala que está cambiando esa postura debido a que la población mayor está en continuo crecimiento. Por otra parte dispone de mayor tiempo libre, lo que les concede la categoría de potenciales clientes de nuevos productos, y finalmente, se incorpora una gran cantidad de nuevos jubilados acostumbrados a la sociedad de consumo, en los que, si bien sus ingresos no serán muy elevados, se convertirán en objetivo de campañas publicitarias específicas para ellos, pues el sector publicitario no podrá prescindir de ese 30\% de potenciales clientes que habría en el 2051.

\subsection{Situación en Andalucía}

Puesto que la muestra se circunscribe a Andalucía y a la RTVA, si comparamos datos de condiciones de vida de esta comunidad y nacionales, según el CIS en 2006 sobre 
condiciones de vida de las personas mayores, la actividad que más realizaron todos o casi todos los días fue ver la televisión (89,8\% en Andalucía, 90,4\% nacional), más del doble que la segunda actividad, que fue oír la radio.

En un informe previo del CIS, de 1998 sobre la soledad en personas mayores, daba como resultado que lo que más hacían cuando se sentían solos era poner la TV o la radio $(28,4 \%)$, seguido de pasear $(22,7 \%)$, y cuando se preguntaba sobre qué actividad habían hecho la última semana, se imponía ver la televisión con un 96,9\%, seguida, a veinticinco puntos, de oír la radio o pasear.

El Instituto de Estadística y Cartografía de Andalucía fija como datos de 2013 una población de 8.440 .300 de los que mayores de 65 serían 1.320.700, el 15,65\%. El mismo Instituto publicó una encuesta de empleo del tiempo en 2009-2010, y en la franja de edad de mayores de 65, veía la tele un $95 \%$ una media de 4 horas y 26 minutos (9 puntos sobre la media)

\subsubsection{Comportamiento de los mayores de Andalucía frente al televisor}

En Andalucía para la medición de audiencias se contabilizan 1.333 .000 mayores de 65 años (408.000 superan los 75), siendo el perfil dominante las mujeres (56,57\%) y la clase social media baja o baja (casi el $45 \%$ ).

Acudiendo al Departamento de Audiencias de Canal Sur y basándonos en los datos que ofrece Kantar Media, empresa especializada en su medición, hemos apreciado que la audiencia media de Canal Sur de enero a julio de 2014, daría un porcentaje del 20,1\% de mayores de 65 años, casi 4,5 puntos más de lo que representa en población. Si nos quedáramos con los que superan los 75 años, la audiencia se elevaría al $26 \%$. En el perfil de los usuarios, las mujeres representan el 21,1\% y la clase media baja o baja el $26,6 \%$.

COMPORTAMIENTO DE LOS MAYORES DE ANDALUCÍA 


\section{FRENTE A LA TELEVISIÓN}

\section{CUOTA DE \\ PANTALLA \\ $\%$ \\ (Datos 1-1- \\ 2014 a 6-7- \\ 2014)}

\begin{tabular}{|c|c|c|c|c|c|c|c|c|c|c|}
\hline TARGET & $\begin{array}{l}\text { C.SU } \\
\text { R }\end{array}$ & $\begin{array}{l}\text { TVE } \\
1\end{array}$ & $\begin{array}{l}\text { TVE } \\
2\end{array}$ & T5 & A3 & $\begin{array}{l}\text { CUA } \\
\text { TRO }\end{array}$ & $\begin{array}{l}\text { LA } \\
\text { SEXT } \\
\text { A }\end{array}$ & $\begin{array}{l}\text { TD } \\
T\end{array}$ & $\begin{array}{l}\text { TEMAT } \\
\text { PAGO }\end{array}$ & $\begin{array}{l}\text { REST } \\
\text { O }\end{array}$ \\
\hline MAYORES EN & & & & & & & & & & \\
\hline $\begin{array}{l}\text { ANDALUCIA } \\
\text { DE } 65 \text { y + años }\end{array}$ & 20,1 & 9,8 & 3,2 & $\begin{array}{r}15, \\
9\end{array}$ & 13,5 & 4,1 & 5,8 & 21 & 4,5 & 2,1 \\
\hline De 65 a 74 años & 17,5 & 10,2 & 3,3 & $\begin{array}{r}15, \\
6\end{array}$ & 13,5 & 4,1 & 6,8 & 21,8 & 5,2 & 2 \\
\hline De 75 y + & 26 & 8,9 & 3 & $\begin{array}{r}16, \\
7\end{array}$ & 13,5 & 4,1 & 3,7 & 19,2 & 2,9 & 2 \\
\hline $\begin{array}{l}\text { Hombres de } 65 \text { y } \\
+\end{array}$ & 18,8 & 10,3 & 3,9 & $\begin{array}{r}12 \\
5\end{array}$ & 12,2 & 4,8 & 7,6 & 21,1 & 6,7 & 2 \\
\hline $\begin{array}{l}\text { Mujeres de } 65 y \\
+\end{array}$ & 21,1 & 9,4 & 2,7 & $\begin{array}{r}18 \\
6\end{array}$ & 14,6 & 3,5 & 4,5 & 20,8 & 2,9 & 1,9 \\
\hline $\begin{array}{l}\text { Clase social } \\
\text { media de } 65 \text { y }+\end{array}$ & 17,5 & 10,7 & 2,9 & $\begin{array}{r}18 \\
1\end{array}$ & 17,3 & 4 & 6,2 & 18,3 & 2,9 & 2,1 \\
\hline
\end{tabular}

Tabla 1: Comportamiento de los mayores andaluces ante la televisión

Fuente: Kantar media

Diferenciando entre la generalidad de las televisiones y Canal Sur, los datos serían: 


\begin{tabular}{|c|c|c|c|c|}
\hline ( TODAS C & ADENAS Y CANA & $\mathrm{IR})-\mathrm{CUO}^{-}$ & Y RA & NG \\
\hline CADENAS & TARGETS & $\begin{array}{l}\text { UNIVERSO } \\
\text { (miles) }\end{array}$ & $\begin{array}{l}\text { CUOTA } \\
\%\end{array}$ & MILES \\
\hline & $\begin{array}{l}\text { MAYORES EN } \\
\text { ANDALUCIA } \\
\text { DE } 65 \text { y + años }\end{array}$ & 1333 & 100 & 344 \\
\hline TOTAL & \begin{tabular}{|l} 
De 65 a 74 años \\
\end{tabular} & 925 & 100 & 238 \\
\hline TODAS LAS & De $75 y+$ & 408 & 100 & 105 \\
\hline TELEVISIONES & Hombres de $65 \mathrm{y}+$ & 578 & 100 & 148 \\
\hline & Mujeres de $65 \mathrm{y}+$ & 754 & 100 & 195 \\
\hline & $\begin{array}{l}\text { Clase social } \\
\text { media de } 65 \mathrm{y}+\end{array}$ & 452 & 100 & 109 \\
\hline & $\begin{array}{l}\text { MAYORES EN } \\
\text { ANDALUCIA } \\
\text { DE } 65 \mathrm{y}+\text { años }\end{array}$ & 1333 & 20,1 & 69 \\
\hline CANAL & De 65 a 74 años & 925 & 17,5 & 42 \\
\hline & De $75 y+$ & 408 & 26 & 27 \\
\hline & Hombres de $65 \mathrm{y}+$ & 578 & 18,8 & 28 \\
\hline & Mujeres de $65 \mathrm{y}+$ & 754 & 21,1 & 41 \\
\hline & $\begin{array}{l}\text { Clase social } \\
\text { media de } 65 \mathrm{y}+\end{array}$ & 452 & 17,5 & 19 \\
\hline
\end{tabular}

Tabla 2: Audiencia de mayores en Andalucía

Fuente: Kantar media 
Observamos, por tanto, que en Andalucía la audiencia de los mayores se concentra en torno a la televisión autonómica con una programación más orientada al consumo familiar. Esta audiencia mayor conlleva un problema a los operadores, el del bajo nivel de reposición de esa audiencia y el descenso del valor de las unidades de venta publicitaria debido al menor potencial como consumidor de este grupo de población, que los deja fuera de las estrategias comerciales de inversión publicitaria. Así, en ocasiones, a pesar de tener altos niveles de audiencia, el valor de la venta publicitaria, los conocidos GRP (Gross Rating Point) desciende considerablemente. Este factor es determinante para que las cadenas comerciales con la publicidad como su principal fuente de financiación minusvaloren a este público.

Si detallamos los programas preferidos de la población superior a 65 años en Andalucía, serían:

SELECCIÓN PROGRAMAS CSTV Y NIVEL
AUDIENCIA
\begin{tabular}{|l|r|}
\hline PROGRAMA & AUDIENCIA $\%$ \\
\hline MISA DE ROMEROS & 47,5 \\
\hline NOTICIAS 1 & 29,7 \\
\hline NOTICIAS PRONVINCIALES 1 & 33,2 \\
\hline LA TARDE AQUÍ AHORA & 28,1 \\
\hline MENUDA NOCHE & 25,7 \\
\hline TOROS PARA TODOS & 35 \\
\hline SE LLAMA COPLA & 31,9 \\
\hline SE LLAMA COPLA (JUNIOR) & 27,8 \\
\hline SALUD AL DIA & 21,9 \\
\hline COMETELO & 23,6 \\
\hline DESTINO ANDALUCIA & \\
\hline
\end{tabular}




\begin{tabular}{|l|r|}
\hline 25 AÑOS GALA ANDALUCÍA 28-F & 28,2 \\
\hline LA BÁSCULA & 19,2 \\
\hline
\end{tabular}

Tabla 3: Programas de Canal Sur y audiencia de mayores en Andalucía

Fuente: Kantar media

Llama la atención que los más seguido sean dos emisiones no regulares, como la Romería del Rocío y la gala del Día de Andalucía. Luego dos informativos (Noticias 1, y su desconexión provincial), y programas relacionados con la tauromaquia. Igualmente destacan el concurso sobre copla y dos presentados por Juan y Medio. Uno de ellos, "La Tarde Aquí y Ahora", no está dirigido específicamente a la población mayor, pero acapara su atención al contar con una sección de personas buscando pareja, entre las que predominan los mayores. El otro programa tiene como actores principales a menores.

Por encima de la media hay otros cuatro espacios; dos de saludables: "Cómetelo" y "Salud al día"; y otros dos vinculados con la Comunidad "Destino Andalucía" y "Este es mi pueblo".

\subsubsection{La tercera edad en los informativos de Canal Sur Televisión}

Ya que la tercera edad pasa muchas horas viendo la televisión, queremos incluir en este estudio el análisis de la imagen que reflejan los informativos sobre este colectivo.

Canal Sur, es la televisión autonómica de Andalucía. Sus índices de audiencia rondan el $10 \%$, y en la actualidad no cuenta con ningún programa de ficción de producción propia, por lo que el análisis se centrará en los informativos. Se agrupan en dos categorías "diarios" y "no diarios", según un doble criterio, la periodicidad y el contenido. Es decir, no sólo dependerá de su frecuencia de emisión, sino también que se ajuste estrictamente a la actualidad. Así, por ejemplo, Andalucía Directo se emite diariamente, pero se incluirá en "no diarios", pues su formato es diferente a los noticiarios. 
Programas "diarios" en Canal Sur son seis: dos informativos matutinos (Primera Hora y Buenos Días Andalucía); uno de sobremesa (Noticias 1); uno de noche (Noticias 2), otro de madrugada (Noticias 3), más otro de noche (La noche al día) que se emite sólo en Canal Sur HD. También se ha incluido uno deportivo (Tododeporte) porque contenía una información relacionada.

En cuanto a los "no diarios" hemos contemplado 16 programas que tienen un carácter divulgativo o de actualidad. Dejamos al margen programas no informativos, como los religiosos, o de entretenimiento.

Basándonos en palabras clave y coordinándolos con los criterios utilizados por el Servicio de Archivo, Documentación y Biblioteca ${ }^{3}$ de Canal Sur, para realizar una búsqueda del material emitido por cualquiera de sus cadenas y acotando el periodo de búsqueda a una temporada televisiva (del 1 de septiembre de 2013 a 30 de junio de 2014) se han introducido los siguientes descriptores:

- ANCIANOS DESASISTIDOS: casos de personas mayores que padecen abandono o están desatendidos por familiares o instituciones.

- AYUDA A DOMICILIO: servicio asistencial, ayuda domiciliaria a personas dependientes (discapacitados, personas mayores, etc.).

- CENTROS ASISTENCIALES: centros de día, residencias para discapacitados, personas dependientes o enfermos con patologías específicas.

\footnotetext{
${ }^{3}$ La consulta se ha podido hacer contando con la colaboración de miembros del Servicio. La solicitud de búsquedas desde fuera de Canal Sur -algo que no es corriente-, hay que tramitarla a través de la Jefa del Servicio, y depende de la complejidad y de la disponibilidad de personal el poder llevarlas a cabo
} 
- ENVEJECIMIENTO ACTIVO: actividades, modos de vida, hábitos y cuidados que contribuyen a mejorar la calidad de vida de las personas mayores.

- GERIATRÍA: especialidades médicas o enfermedades

- JUBILACIONES: jubilados; jubilaciones anticipadas, bajas voluntarias.

- PENSIONES DE JUBILACIÓN: pensionistas.

- PERSONAS MAYORES: grupos de edad

- RESIDENCIAS DE ANCIANOS: centros de día, residencias asistidas, geriátricos, centros asistenciales.

- TERCERA EDAD: información general sobre viejos, ancianos y personas mayores

- VEJEZ Y ENVEJECIMIENTO: pérdida de calidad de vida a causa de enfermedades o de achaques propios de la edad.

Con estos criterios, los resultados para la temporada 2013/2014 han sido 366 documentos, entendiendo por documento aquella información audiovisual emitida, analizada y registrada por los documentalistas de Canal Sur. Cuando se ha repetido en distintos informativos (por ejemplo, en Noticias 1 y repetido en Noticias 2), no se ha contabilizado, pero sí cuando se altera su forma o se rehace la información aunque sea dentro del mismo informativo, por ejemplo, con unas colas al inicio del programa, y después como noticia completa. También señalar que el estudio se ha hecho con datos regionales y con la desconexión de Sevilla, ya que las desconexiones de las otras 7 provincias andaluzas no están centralizadas para su búsqueda. 
De los 246 documentos de "diarios" hemos diferenciado entre los distintos espacios, y por contenidos.

\begin{tabular}{|l|c|}
\hline \multicolumn{2}{|c|}{ PRESENCIA EN NOTICIAS DE } \\
PROGRAMAS INFORMATIVOS DIARIOS \\
\hline INFORMATIVO & N $^{\mathbf{0}}$ veces tratado \\
\hline Primera Hora & 5 \\
\hline Buenos Días Andalucía & 33 \\
\hline Noticias 1 & 75 \\
\hline Desconexión Prov. N1 & 16 \\
\hline Noticias 2 & 56 \\
\hline Desconexión Prov. N2 & 25 \\
\hline Noticias 3 & 7 \\
\hline La Noche al Día & \\
\hline Tododeporte & \\
\hline
\end{tabular}

Tabla 4: Presencia de Tercera Edad en programas diarios

Fuente: Departamento Archivo y Documentación de Canal Sur TV

El formato elegido para dar la noticia ha sido: 109 colas (locución del presentador-a sobre imágenes), 101 vídeos elaborados por redactor-a, 32 sólo declaraciones y 4 conexiones en directo. 
Por temática hemos considerado una agrupación por contenidos con las siguientes categorías:

- ENVEJECIMIENTO ACTIVO: realización de actividades diversas como estudiar en la universidad, colaborar con ONG o hacer deporte.

- SUCESOS: como víctimas de estafas, robos o atracos.

- HOMENAJES: por longevidad, por centenarios, o por desempeño de una actividad durante muchos años.

- PENSIONES-JUBILACIÓN: muy mediatizado por el debate sobre la elevación de la edad de jubilación, recorte de pensiones.

- ASISTENCIAL: relacionados con la teleasistencia, dependencia, centros de atención, residenciales o no. Se excluyen los conflictos laborales en los mismos.

- CRISIS ECONÓMICA: exclusión, sustento de la familias, desahucios.

- CAMPAÑAS DE CONCIENCIACIÓN: dirigidas específicamente a este colectivo o en las que participan.

- ENFERMEDADES O ASUNTOS MÉDICOS: tratados desde el punto de vista geriátrico.

- SOLEDAD: situación que padece una parte de la tercera edad. 
- PROTESTAS: se recogen los conflictos por cierres de centros de la tercera edad y las movilizaciones laborales relacionados con impagos en residencias o en dependencia.

- OTROS: de contenido diverso, relacionados con la esperanza de vida, o con encuestas de opinión, o participación en listas electorales, cooperativas o memoria histórica.

Tabla 5: Temática por programa informativo diario Fuente: Elaboración propia con datos Dpto. Archivo y Documentación CSTV

En las 120 apariciones en "no diarios", se excluyen programas que se hacen eco de la tercera edad. De los 16 estudiados en 7 no hay referencias. En Andalucía Directo, Más que Noticias, Destino Andalucía, Parlamento Andaluz, Tierra y Mar, Solidarios, Salud al

\begin{tabular}{|l|l|l|l|l|l|l|l|l|l|l|}
\hline Temas & PH & BD & N1 & DN1 & N2 & DN2 & N3 & ND & TD & TOTAL \\
\hline Jubilac.-pensión & 2 & 14 & 23 & 0 & 22 & 0 & 6 & 18 & 0 & $\mathbf{8 5}$ \\
\hline Asistencia-dep. & 2 & 4 & 12 & 5 & 8 & 3 & 1 & 4 & 0 & $\mathbf{3 9}$ \\
\hline Enveje. Activo & 0 & 0 & 7 & 3 & 6 & 9 & 0 & 0 & 1 & $\mathbf{2 6}$ \\
\hline Protestas & 0 & 5 & 5 & 6 & 2 & 7 & 0 & 0 & 0 & $\mathbf{2 5}$ \\
\hline Sucesos & 0 & 2 & 8 & 1 & 5 & 0 & 0 & 1 & 0 & $\mathbf{1 7}$ \\
\hline Enfermedad & 0 & 0 & 11 & 0 & 5 & 1 & 0 & 0 & 0 & $\mathbf{1 7}$ \\
\hline Dificultad Eco. & 1 & 2 & 3 & 0 & 3 & 0 & 0 & 4 & 0 & $\mathbf{1 3}$ \\
\hline Homenajes & 0 & 2 & 0 & 1 & 0 & 4 & 0 & 0 & 0 & $\mathbf{9}$ \\
\hline Campaña Conc. & 0 & 1 & 3 & 0 & 1 & 1 & 0 & 1 & 0 & $\mathbf{7}$ \\
\hline Soledad & 0 & 0 & 1 & 0 & 0 & 0 & 0 & 0 & 0 & $\mathbf{1}$ \\
\hline Otros & 0 & 3 & 2 & 0 & 4 & 0 & 0 & 0 & 0 & $\mathbf{9}$ \\
\hline TOTAL & $\mathbf{5}$ & $\mathbf{3 3}$ & $\mathbf{7 5}$ & $\mathbf{1 6}$ & $\mathbf{5 6}$ & $\mathbf{2 7}$ & $\mathbf{7}$ & $\mathbf{2 8}$ & $\mathbf{1}$ & $\mathbf{2 4 6}$ \\
\hline
\end{tabular}


Día, Aldea Global y Los Reporteros sí.

\begin{tabular}{|l|c|}
\hline \multicolumn{2}{|c|}{ PRESENCIA DE NOTICIAS EN PROGRAMAS } \\
INFRMATIVOS NO DIARIOS \\
\hline PROGRAMA & N $^{\text {de veces tratado }}$ \\
\hline Andalucía Directo (AD) & 81 \\
\hline Más que Noticias (MQN) & 18 \\
\hline Aldea Global (AG) & 5 \\
\hline Salud al Día (SD) & 5 \\
\hline Solidarios (SOL) & 4 \\
\hline Los Reporteros (REP) & 3 \\
\hline Destino Andalucía (DA) & 1 \\
\hline Tierra y Mar (TM) & \\
\hline Parlamento Andaluz (PA) & \\
\hline
\end{tabular}

Tabla 6: Presencia de Tercera Edad en programas no diarios

Fuente: Departamento de Archivo y Documentación de CSTV

Aunque el propósito era mantener las categorías de los informativos diarios, algunas varían por la diferencia de contenidos. Por ejemplo, el retraso en la edad de jubilación era destacado en los telediarios, pero es irrelevante en los "no diarios", y por el contrario, la diversión o la movilidad no aparece en los "diarios".

- ENVEJECIMIENTO ACTIVO: realización de actividades como estudiar en la universidad, colaborar con ONG o cuidar de nietos. 
- SUCESOS: donde son víctimas de estafas, robos o atracos.

- HOMENAJES: por longevidad, por centenarios, o por el desempeño de una actividad durante muchos años.

- MOVILIDAD: derivadas de riesgo cuando se desplazan, como riesgo a caídas.

- DIVERSIÓN: actividades lúdicas.

- ASISTENCIAL: relacionados con teleasistencia, dependencia o centros de atención, residenciales o no. Se incluyen conflictos laborales en los mismos.

- ECONOMÍA: pensiones, exclusión, apoyo familiar, impuestos de retornados, o patrimonio.

- NUEVAS TECNOLOGÍAS: utilización que hacen de las mismas. Suelen ser curiosidades.

- CAMPAÑAS DE CONCIENCIACIÓN: dirigidas específicamente a este colectivo o en las que participan.

- ENFERMEDADES O ASUNTOS MÉDICOS: tratados desde el punto de vista geriátrico.

- SOLEDAD: situación que padece una parte de la tercera edad. 
- OTROS: relacionados con la esperanza de vida, o encuestas opinando sobre la abdicación del rey, o el recuerdo del asesinato de J.F.Kennedy.

Tabla 7: Temática por programa informativo no diario

Fuente: Elaboración propia con datos Dpto. Archivo y Documentación CSTV

\section{DISCUSIÓN Y CONCLUSIONES}

A tenor de los datos estudiados, la población mayor es la consumidora principal de la televisión, pero se convierte en una protagonista secundaria. Está infrarrepresentada.

Aunque hay noticias que se repiten en varios informativos el mismo día, podemos

\begin{tabular}{|l|l|l|l|l|l|l|l|l|c|c|}
\hline & AD & MQN & AG & SD & SOL & REP & DA & TM & PA & TOTAL \\
\hline Envej. Activo & 19 & 0 & 1 & 0 & 3 & 0 & 0 & 1 & 0 & $\mathbf{2 4}$ \\
\hline Homenajes & 16 & 0 & 0 & 0 & 0 & 0 & 0 & 0 & 0 & $\mathbf{1 3}$ \\
\hline Sucesos & 12 & 0 & 1 & 0 & 0 & 0 & 0 & 0 & 0 & $\mathbf{1 3}$ \\
\hline Economía & 4 & 6 & 0 & 0 & 0 & 1 & 0 & 0 & 0 & $\mathbf{1 1}$ \\
\hline Diversión & 3 & 2 & 0 & 4 & 0 & 0 & 2 & 0 & 0 & $\mathbf{1 1}$ \\
\hline Enfermedad & 0 & 4 & 2 & 0 & 1 & 1 & 0 & 0 & 1 & $\mathbf{9}$ \\
\hline C. Conciencia. & 7 & 1 & 0 & 0 & 0 & 0 & 0 & 0 & 0 & $\mathbf{8}$ \\
\hline Asistencial & 5 & 0 & 0 & 1 & 0 & 1 & 0 & 0 & 0 & $\mathbf{7}$ \\
\hline Movilidad & 7 & 0 & 0 & 0 & 0 & 0 & 0 & 0 & 0 & $\mathbf{7}$ \\
\hline N. Tecnología & 0 & 4 & 1 & 0 & 0 & 0 & 0 & 0 & 0 & $\mathbf{5}$ \\
\hline Soledad & 5 & 0 & 0 & 0 & 0 & 0 & 0 & 0 & 0 & $\mathbf{5}$ \\
\hline Otros & 3 & 1 & 0 & 0 & 0 & 0 & 0 & 0 & 0 & $\mathbf{4}$ \\
\hline & $\mathbf{8 1}$ & $\mathbf{1 8}$ & $\mathbf{5}$ & $\mathbf{5}$ & $\mathbf{4}$ & $\mathbf{3}$ & $\mathbf{2}$ & $\mathbf{1}$ & $\mathbf{1}$ & $\mathbf{1 2 0}$ \\
\hline & & & & & & & & & & \\
\hline
\end{tabular}


deducir que los mayores son escasamente tratados para ser casi el $18 \%$ de la población, ya que se tratan en los informativos de media una vez al día, y eso considerando todos los programas informativos de la televisión andaluza.

Cabe destacar que es una audiencia más fiel a las televisiones públicas que a las privadas. Sin embargo, no suele recibir una atención especial pues interesa como número que engrosa la audiencia, pero no como consumidores.

Existe una relación directa entre la atención que prestan las televisiones públicas a este colectivo frente a los operadores privados más interesados por la venta de publicidad por lo que se percibe como un target con poco poder adquisitivo.

En los informativos diarios de la televisión andaluza cuando más aparecen noticias relacionadas con los mayores es en sobremesa y noche, y predominan los asuntos en los que juegan un papel pasivo; incluso en algunas noticias sobre envejecimiento activo no asumen el protagonismo, sino la recomendación de practicarlo.

El rol que desempeñan suele ser secundario.

En los programas "no diarios", tienen más esa posición que les hace ser protagonistas, como en casos de envejecimiento activo, o los homenajes. Aquí la particularidad sí destaca sobre la colectividad.

Como audiencia presta más atención a los programas que se refieren de su tierra o asuntos que se vinculan a ella, como romerías, toros, pueblos, o los informativos.

Igualmente, se interesan más por los programas de entretenimiento simple.

Asistimos a un cambio en el paradigma que se ha mantenido en los últimos treinta años entre la televisión y personas mayores. En la actualidad vemos una actitud pasiva por parte de nuestros mayores, fruto de elementos culturales, formativos y nivel de ingresos. Sin embargo, podemos intuir que en los próximos años se 
incorporará una generación con mayor nivel de formación y manejo de las nuevas tecnologías, y por tanto, más activa ante los televisores smart e Internet, lo que provocará nuevas formas de consumir televisión. Asimismo, los grandes operadores privados se verán abocados a prestar atención a esta bolsa de población que puede llegar al $30 \%$ en poco tiempo, y por tanto, será un target interesante para las centrales de compra publicitaria, y como consecuencia para los operadores.

Es necesario una mayor conciencia por parte de los operadores televisivos de la importancia de la tercera edad como grupo social y concederles más presencia protagonista en todo tipo de programas, informativos y de ficción.

\section{REFERENCIAS}

Ageing characterises the demographic perspectives of the European societies. EUROSTAT, 2010. Recuperado de http:/ / polennu.dk/sites/default/ files/Eurostat \%20befolkningsprognose $\% 202008$ -2060.pdf. Consultado el: 14/10/2014.

Aguaded, J. (1999): Convivir con la televisión. Familia, educación y recepción televisiva. Barcelona. Paidós

CIS. Condiciones de vida de las personas mayores Resultados Total nacional y Andalucía. Recuperado de http://www.cis.es/cis/opencm/ES/1_encuestas/estudios/ver.jsp?estudio=7740 $\underline{\text { \&cuestionario }=8954 \& \text { muestra }=14085}$. Consultado el: 06/09/2014.

CIS: La Soledad en las personas mayores. Estudio n 2.279 Febrero-Marzo 1998. Recuperado de http://www.cis.es/cis/export/sites/default/Archivos/Marginales/2260_2279/2279/Es2279mar.pdf. Consultado el: 16/09/2014. 
Consejo Audiovisual de Andalucía. Informe sobre la presencia de los colectivos necesitados de mayor protección en los informativos de las televisiones públicas de Andalucía.

Recuperado

de

http://www.consejoaudiovisualdeandalucia.es/sites/default/files/informes/inf orme_colectivo_de_mayor_proteccion_y_votos_particulares.pdf. Consultado el: 28/09/2014.

Díaz Casanova, M. (1989). Envejecimiento de la población y conflicto entre generaciones. Reis, 45, 85-113

Ferrés i Prats, J. (2005). La familia frente al televisor:¿víctima o culpable?. Comunicar, 25, 237-239.

Freire, P. (1997): A la sombra de este árbol. Barcelona: El Roure

FUNDAEC: El baròmetre de la comunicació i la cultura. Recuperado de http:// www.fundacc.org/fundacc/es/noticies/fitxa.html?param10=9277\&ed=16. Consultado el: 23/09/2014.

Fundación Edad y Vida. (2009): Los hábitos de compra y consumo de las personas mayores. Madrid. Senda Editorial, S.A.

Instituto Nacional de Estadística. Esperanza de vida. Recuperado de http:/ / www.ine.es/ss/Satellite?L=es_ES\&c=INESeccion_C\&cid=1259926380048\& $\mathrm{p}=1254735110672 \&$ pagename=ProductosYServicios/PYSLayout. Consultado el: $10 / 09 / 2014$.

Instituto de Estadística y Cartografía de Andalucía. Encuesta de Empleo del Tiempo 2009-2010. Recuperado de http://www.ieca.junta-andalucia.es/eet/index.htm. Consultado el: 03/10/2014. 
Instituto de Estadística y Cartografía de Andalucía. Población Andalucía. Recuperado de http://www.ieca.junta-andalucia.es/temas/tema02.html. Consultado el: 03/10/2014.

LANZIERI, Giampaolo (2013) Towards a 'baby recession' in Europe? Differential fertility trends during the economic crisis. EUROSTAT. Recuperado de http://europa.eu/epc/pdf/economic_and_budgetary_projections_for_the_27_eu _member_states_en.pdf. Consultado el 16/10/2014.

López, J.A. \& Cuenca, F.A. (2005). Ficción televisiva y representación generacional: modelos de tercera edad en las series nacionales. Comunicar, 25, 231-236. DOI: http://dx.doi.org/10.3916/C37-2011-03-05

Medrano, C., Cortés, A., Aierbe, A. \& Orejudo, S. (2010). Los programas y características de los personajes preferidos en el visionado de la televisión: diferencias evolutivas $\mathrm{y}$ de sexo. Taylor $\mathcal{E}$ Francis online, 22-1. DOI: http://dx.doi.org/10.1174/113564010790935196. Consultado el: 03/11/2014.

Population. $\quad 2010 . \quad$ RUROSTAT, http:/ / ec.europa.eu/eurostat/help/new-eurostat-website. Consultado 30/09/2014.

Requejo Osorio, A. (1998). Sociedad del Aprendizaje y Tercera Edad. Teoría de la Educación, 10, 145-167.

Rincón, O. (2006). Narrativas mediáticas. Barcelona: Gedisa.

Terceiro, J. B. (1996): Sociedad digital. Del homo sapiens al homo digitalis. Madrid: Alianza Editorial. 
Torres, M. (2006). Actitudes y motivaciones de las personas mayores hacia su desarrollo personal. Madrid: Secretaría General Técnica Consejería de Servicios Sociales.

Sánchez Vera, P. (1996). Tercera y cuarta edad en España desde la perspectiva de los hogares. Reis, 73, 57-79.

Vaca Bercayes, R. (2004). El ojo digital. Madrid: Fundación Exlibris

World Health Organization, (2002): Active Ageing: A Policy Framework. Ginebra (Suiza). Organización Mundial de la Salud

\section{AUTORES}

\section{Agustín Olmo López:}

Combina el ejercicio práctico de la profesión periodística con la docencia. Es Doctor en Periodismo por la Universidad Complutense de Madrid y trabaja como periodista en Canal Sur TV especializado en programas de reportajes. Es también profesor asociado en la Facultad de Comunicación de la Universidad de Sevilla en Técnicas y Procesos del Periodismo Audiovisual y en Cibercultura. Sus investigaciones tienen que ver con audiencias y televisión y con temas europeos.

\section{José Antonio Navarro Moreno:}

Cuenta con un currículum que busca el equilibrio entre actividades docentes, investigadoras y laborales. Desde la perspectiva académica ha tenido relación con tres Universidades: Complutense, Autónoma de Barcelona y la de Sevilla. Es precisamente, en esta última, en la que trabaja como profesor asociado. Doctor en comunicación con la tesis "La Televisión local en Andalucía". En su faceta de investigador ha estado vinculado a varios grupos, tanto en la Universidad de Sevilla, como de la Autónoma de Barcelona. En el año 2009, lidera el proyecto de investigación, "Foro Andaluz de Comunicación Europea". En su trayectoria laboral 
ha sido periodista en "Canal Sur Televisión" desde 1990. En este medio ha realizado tareas como el diseño de programas o la realización de documentales. Entre su bibliografía se encuentra "La televisión Local en Andalucía, la nueva Comunicación". (1999) o "10 años de RTVA". (2000). 